\title{
Efficiency of Barley Bran and Oat Bran in Ameliorating Blood Lipid Profile and the Adverse Histological Changes in Hypercholesterolemic Male Rats
}

\author{
Haddad A. El Rabey, ${ }^{1,2}$ Madeha N. Al-Seeni, ${ }^{1}$ and Hanan M. Amer ${ }^{3}$ \\ ${ }^{1}$ Biochemistry Department, Faculty of Science, King Abdulaziz University, Jeddah, Saudi Arabia \\ ${ }^{2}$ Bioinformatics Department, Genetic Engineering and Biotechnology Research Institute, Minufiya University, P.O. Box 79, \\ Sadat City, Egypt \\ ${ }^{3}$ Badreddin Clinic, Jeddah, Saudi Arabia \\ Correspondence should be addressed to Haddad A. El Rabey; elrabey@hotmail.com
}

Received 19 March 2013; Revised 1 June 2013; Accepted 11 July 2013

Academic Editor: Anne Pihlanto

Copyright $\odot 2013$ Haddad A. El Rabey et al. This is an open access article distributed under the Creative Commons Attribution License, which permits unrestricted use, distribution, and reproduction in any medium, provided the original work is properly cited.

\begin{abstract}
The efficiency of oat bran and barley bran in lowering the induced hyperlipidemia and hypercholesterolemia in blood of male Albino rats (Rattus rattus) was studied. Twenty rats were divided into four groups each consisted of five rats and fed the specified test diets for eight weeks. The first group (G1) is the negative group which was fed basal diet, the second group (G2) was fed $1.0 \%$ cholesterol, was the third group (G3) fed 1.0\% cholesterol and $10 \%$ oats bran, and the fourth group (G4) was fed $1.0 \%$ cholesterol and $10 \%$ barley bran. Feeding rats on $1 \%$ cholesterol significantly increased serum total cholesterol, low density lipoprotein, and very low density lipoprotein and triglyceride and decreased serum high density lipoprotein. Furthermore, enzyme activity of alanine aminotransferase, aspartate aminotransferase, and alkaline phosphatase was increased, and lipid peroxide was increased, whereas catalase and glutathione-S-transferase were decreased. Kidney functions parameters in the cholesterol supplemented group were elevated compared with the negative control. In addition, histological alteration in kidney, liver, heart, and testes was observed, compared with the negative control. Hypercholesterolemic rats supplemented with oat bran and barley bran showed significant decrease in lipid parameters, significant increase in high density lipoprotein-cholesterol, improved antioxidant enzyme, and improved histopathology of kidney, liver, heart, and testes. In conclusion, both oat bran and barley bran had protective effects against induced hyperlipidemia and improved histological alterations. Oat bran appeared more efficient than barley bran in lowering the lipid profile levels in hypercholesterolemic rats.
\end{abstract}

\section{Introduction}

Dietary or pharmacological reduction of total and low density lipoproteins (LDL) decreases the risk of coronary heart diseases (CHD) [1]. LDL is the major atherogenic lipoprotein, and numerous clinical trials have shown the efficacy of lowering low density lipoproteins-cholesterol (LDL-C) for reducing CHD risk [2]. Consumption of diets rich in whole grains has been reported to have beneficial health effects such as reducing the risk of cancer, cardiovascular disease, and noninsulin-dependent diabetes mellitus. These results have been attributed to the effects of the fiber content of whole-grain foods on risk factors of these diseases, including blood glucose, insulin, and cholesterol [3]. Numerous studies have demonstrated that whole grains that are high in soluble fiber, such as oats and barley, are more effective in lowering blood cholesterol than grains in which fibers are predominantly insoluble, such as wheat or rice $[4,5]$.

Increasing dietary fiber, which is a variety of plant substances that are resistant to digestion by human gastrointestinal enzymes, has been used for cholesterol reduction $[6,7]$. Moreover, dietary fibers can be classified into two major groups depending on their solubility in water in human body, and the structural or matrix fibers (lignins, cellulose, 
and some hemicelluloses) are insoluble, whereas the natural gel-forming fibers (pectins, gums, mucilages, and the remainder of the hemicelluloses) are soluble. Studies have focused on soluble fibers such as oats, psyllium, pectin, barley, and guar gum, and qualitative reviews suggested that these fibers lower total and LDL-C $[6,7]$.

$\beta$-glucan is a nonstarch polysaccharide composed of beta-(1-4)-linked glucose units separated every 2-3 units by beta-(1-3)-linked glucose [8]. This soluble fiber, which is found in oat and barley, has a potential to reduce LDL-C through the increase in intestinal viscosity that may lower cholesterol absorption, although reduced cholesterol absorption was not found in other studies $[8,9]$.

Oat is an important source for water-soluble fibers, and the beneficial effects of oat products on the lipoprotein profile are ascribed to their soluble fiber compound, $\beta$-glucan [10]. In addition, oat is a source of antioxidants, such as tocols and various phenolic compounds [11]. Oat antioxidants have been reported to inhibit low-density lipoprotein oxidation and promote scavenging of reactive oxygen species $[12,13]$. There are many studies indicating the efficacy of oat bran in reducing total cholesterol (TC) and LDL-C concentrations while either increasing or having no effect on plasma HDL-C concentrations in humans $[10,14,15]$.

Barley, like oat, is a rich source of the soluble fiber $\beta$ glucan, which has been shown to significantly lower LDL-C [16]. The effects of barley $\beta$-glucan on cardiovascular and diabetic risk were reviewed [17]. Concentrated $\beta$-glucan extracts with high or low molecular weight added to food products were reported efficiently to lower LDL-C in hyperlipidaemic subjects with and without the metabolic syndrome [18]. The consumption of barley and its products might reduce many risk factors associated with the metabolic syndrome, diabetes, hypertension, and dislipidemia [19].

The purpose of this study is testing the efficiency of barley bran and oat bran (as $\beta$-glucan containing nutrients) in lowering blood cholesterol levels and ameliorating the histological changes (resulted from hypercholesterolemia) of kidney, liver, heart, and testes in male rats.

\section{Materials and Methods}

2.1. Animals and Housing Conditions. Twenty male Albino Wister rats "Rattus rattus" weighing about 140-160 g were obtained from King Fahd Medical Research Center, King Abdulaziz University, Jeddah, Saudi Arabia. Rats were housed five per polycarbonate cages. Cages, bedding, and glass water bottles (equipped with stainless steel sipper tubes) were replaced twice per week. Stainless steel feed containers were changed once per week. Oats and barley were obtained from a Grain Silos and Flour Mills. The seeds were milled and then sieved to get the bran. The animals were kept at room temperature $\left(25 \pm 5^{\circ} \mathrm{C}\right)$ with a natural lighting cycle (12 hours), fed a standard basal diet [20], and kept under observation for 2 weeks before the start of the experiment to exclude any undercurrent infection.

2.2. Experiment Design. The animals were then divided randomly into four groups each of 5 rats as follows: group 1 (G1): fed normal diet, group 2 (G2): fed 1.0\% w/w cholesterol expure, from Oxford Laboratory Reagent Company (Egypt) in diet to induce hypercholesterolemia [21], group 3 (G3): fed cholesterol as in (G2) and supplemented with $10 \%$ oat bran [22], and group 4 (G4): fed cholesterol as in (G2) and supplemented with $10 \%$ barley bran to be equal to that of oat bran.

The trial period of the current study was 8 weeks because it has been proven to be the most efficient period in causing hypercholesterolemia $[23,24]$. At the 8 th week blood samples were collected from all rats after anaesthetizing them by dimethyl-ether, and then blood samples were collected from the ophthalmic orbital sinus [25]. It is generally accepted that the most reliable data on blood lipid metabolism can be obtained from fasting animals, 14-16 hours after their last feeding. Therefore, food was removed from the cages at 6 p.m. a day before blood samples were drawn, and the samples were collected at 9 a.m. the next day.

2.3. Biochemical Tests. Blood was collected in EDTA tube for $\mathrm{CBC}$ analysis and in plain tubes for chemistry analyses. Serum was obtained by blood samples centrifugation at $1000 \mathrm{rpm}$ for $10 \mathrm{~min}$ at room temperature and then stored at $-20^{\circ} \mathrm{C}$ until analysis was performed. All biochemical analyses (lipid profile tests "sTC, sTG, sLDL-C, sHDL-C, and sVLDLC," liver enzymes "AST, ALT, and ALP," and kidney function parameters "creatinine, urea, and uric acid") were achieved using the specified kits from Gesellschaft for Biochemical and Diagnostic (Germany) according to the instructions of the suppliers. At the end of the experiment and after collection of blood, anaesthetized animals were scarified by cervical dislocation. The abdomen was opened, and the organs were rapidly excised. The left kidney was quickly rinsed in isotonic saline and dried on a piece of filter paper. One hundred gram of kidney tissue was homogenized in ice-cold saline using a glass homogenizer. The homogenate was then diluted with the homogenization medium to ultimately yield $10 \%(\mathrm{v} / \mathrm{v})$. Biodiagnostic Chemical Company (Egypt) kits were used in estimating GST [26], catalase [27], and lipid peroxide [28] in the kidney homogenate according to the instructions of the suppliers.

2.4. Histopathological Investigations. Liver, heart, right kidney, and testis were washed in sterile saline and fixed in $10 \%$ neutral formalin for histopathological studies. The target organs were then dehydrated in gradual ethanol (50-99\%), cleared in xylene, and embedded in paraffin. Sections were prepared and then stained with hematoxylin and eosin (H\&S) dye for microscopic investigation [29]. The stained sections were examined and photographed under a light microscope.

2.5. Statistical Analysis. All data were analyzed using the SPSS (Statistical Program for Sociology Scientists) Statistics Version 17.0 for computing the mean values, the standard errors (SE), and test of significance ( $t$-test).

\section{Results}

3.1. The Effect of Tested Diets on Lipid Profile. The effect of oat bran and barley bran supplementation, for 8 weeks on 
TABLE 1: Effect of oat bran and barley bran supplementation for 8 weeks on serum lipids in rats with induced hypercholesterolemia.

\begin{tabular}{|c|c|c|c|c|c|}
\hline Parameters & Statistics & G1 & G2 & G3 & G4 \\
\hline \multirow{2}{*}{ s.TC (mg \%) } & Mean \pm SE & $92.70 \pm 0.95$ & $119.46 \pm 1.10$ & $67.80 \pm 1.98$ & $73.64 \pm 3.93$ \\
\hline & $t$-test & & $-13.46^{* * *}$ & $20.02^{* * *}$ & $15.15^{* * *}$ \\
\hline \multirow{2}{*}{ s.T.G (mg/dL) } & Mean \pm SE & $93.34 \pm 5.7$ & $114.26 \pm 1.67$ & $55.52 \pm 2.41$ & $62.06 \pm 6.44$ \\
\hline & $t$-test & & $-2.92^{* *}$ & $16.87^{* * *}$ & $7.79^{* * *}$ \\
\hline \multirow{2}{*}{ s.HDLc (mg/dL) } & Mean \pm SE & $13.40 \pm 0.86$ & $8.34 \pm 0.56$ & $18.22 \pm 1.24$ & $18.98 \pm 0.94$ \\
\hline & $t$-test & & $5.94^{* * *}$ & $-9.12^{* * *}$ & $-10.92^{* * *}$ \\
\hline \multirow{2}{*}{ s.VLDLc (mg/dL) } & Mean \pm SE & $17.45 \pm 1.37$ & $20.17 \pm 1.67$ & $11.10 \pm 0.48$ & $12.40 \pm 1.28$ \\
\hline & $t$-test & & $-1.58 \mathrm{NS}$ & $5.53^{* * *}$ & $2.94^{* *}$ \\
\hline \multirow{2}{*}{ s.LDLc (mg/dL) } & Mean \pm SE & $58.93 \pm 1.54$ & $88.12 \pm 3.35$ & $38.48 \pm 2.87$ & $42.26 \pm 4.28$ \\
\hline & $t$-test & & $-6.91^{* * *}$ & $10.59^{* * *}$ & $8.55^{* * *}$ \\
\hline
\end{tabular}

Significant differences with controls calculated by paired sample $t$-test. ${ }^{*}$ means significant at $P<0.05,{ }^{* *}$ means highly significant at $P<0.01,{ }^{* * *}$ means very high significant at $P<0.001$, and NS means nonsignificant.

TABLE 2: Effect of oat bran and barley bran supplementation for 8 weeks on liver enzymes in rats with induced hypercholesterolemia.

\begin{tabular}{lccccc}
\hline Parameters & Statistics & G1 & G2 & G3 & G4 \\
\hline \multirow{2}{*}{ ALT (U/L) } & Mean \pm SE & $32.84 \pm 2.16$ & $38.46 \pm 2.51$ & $28.56 \pm 3.15$ & $37.34 \pm 5.18$ \\
& $t$-test & & $-2.34^{*}$ & $3.62^{*}$ & $-2.66^{*}$ \\
AST (U/L) & Mean \pm SE & $206.20 \pm 19.1$ & $263.02 \pm 26.60$ & $174.80 \pm 6.74$ & $206.80 \pm 16.79$ \\
& $t$-test & & $1.19 \mathrm{NS}$ & $-0.44 \mathrm{NS}$ & $-1.26 \mathrm{NS}$ \\
ALP (U/L) & Mean \pm SE & $182.60 \pm 25.23$ & $294.20 \pm 37.66$ & $189.20 \pm 35.13$ & $184.20 \pm 20.60$ \\
& $t$-test & & $4.88^{* * *}$ & $-0.07 \mathrm{NS}$ & $1.09 \mathrm{NS}$ \\
\hline
\end{tabular}

Significant differences with controls calculated by paired sample $t$-test. ${ }^{*}$ means significant at $P<0.05,{ }^{* *}$ means highly significant at $P<0.01,{ }^{* * *}$ means very high significant at $P<0.001$, and NS means nonsignificant.

serum lipids in rats with induced hypercholesterolemia is illustrated in Table 1. As shown, the mean values of s.TC, s.TG, s.LDL-C, and serum very low density lipoproteincholesterol (s.VLDL-C) in the positive control were higher than that of the negative control, whereas the serum HDL$\mathrm{C}$ was decreased. The differences were highly significant $(P<$ 0.001), in s.TC, S.TG, s.LDL-C, and HDL-C compared with that of the negative control. In G3 and G4, the mean values of the s.TC, s.TG, S.LDL-C, and s.VLDL-C were lower than that of the positive control. The differences were highly significant $(P<0.001)$ compared with the positive control.

3.2. The Effect of Tested Diet on Liver Enzymes. Table 2 shows the effect of oat bran and barley bran supplementation for 8 weeks on liver enzymes in rats with induced hypercholesterolemia. The mean values of ALT, AST, and ALP in the positive control were higher than that of the negative control. The differences were significant $(P<0.05)$ in case of ALT, nonsignificant in case of AST, and highly significant $(P<0.001)$ in case of ALP. In G3 and G4, the mean values of ALT, ALP, and AST were lower than that of the positive control.

3.3. Effect of the Tested Diets on Kidney Function. The effect of barley bran and oat bran supplementation for 8 weeks on kidney function in rats with induced hypercholesterolemia is given in Table 3. The mean values of serum uric acid and urea in the positive control were higher than that of the negative control. The differences were significant $(P<0.05)$ in case of uric acid and highly significant $(P<0.01)$ in case of urea, compared with the negative control. While the values of uric acid and urea in G3 and G4 were lower than that of the positive control, differences were nonsignificant in case of uric acid while being highly significant $(P<0.01)$ in case of urea compared with the positive control. The mean values of creatinine in the positive control were nonsignificantly higher than that of the negative control, while in G3 and G4, the mean values were lower than that of the positive control.

3.4. Effect of the Tested Diets on GST, Catalase, and Lipid Peroxide. The effect of oat bran and barley bran supplementation for 8 weeks on catalase, GST, and lipid peroxide in kidney homogenate of rats with induced hypercholesterolemia is shown in Table 4. The mean values of GST in the positive control were lower than that of the negative control. While the mean values of catalase and lipid peroxide were higher than that of the negative control, the differences were highly significant $(P<0.001)$ in case of GST and catalase compared with the negative control. In G3 and G4, the mean values of GST and catalase were higher than that of the positive control. While the mean values of lipid peroxide were lower than that of the positive control, the differences of catalase mean values in G3 and G4 were highly significant $(P<0.001)$, whereas differences in mean values of lipid peroxide in G4 were significant $(P<0.05)$ compared with the positive control.

\subsection{Histopathological Investigation}

3.5.1. Kidney. Normal renal tissues of control group is shown in Figure 1(a). Normal renal structure with regulated nuclear 
TABLE 3: Effect of oat bran and barley bran supplementation for 8 weeks on kidney function in rats with induced hypercholesterolemia.

\begin{tabular}{lccccc}
\hline Parameters & Statistics & G1 & G2 & G3 & G4 \\
\hline Uric acid mg/dL & Mean \pm SE & $1.96 \pm 0.15$ & $2.48 \pm 0.22$ & $1.94 \pm 0.13$ & $2.32 \pm 0.16$ \\
& $t$-test & & $-2.58^{*}$ & $2.12 \mathrm{NS}$ & $1.24 \mathrm{NS}$ \\
Creatinine umol/L & Mean $\pm \mathrm{SE}$ & $0.36 \pm 0.06$ & $0.38 \pm 0.05$ & $0.34 \pm 0.05$ & $0.34 \pm 0.05$ \\
& $t$-test & & $-0.34 \mathrm{NS}$ & $-0.25 \mathrm{NS}$ & $0.23 \mathrm{NS}$ \\
Urea umol/L & Mean \pm SE & $38.20 \pm 1.06$ & $43.00 \pm 0.70$ & $37.00 \pm 1.58$ & $37.20 \pm 1.49$ \\
& $t$-test & & $-4.49^{* *}$ & $4.04^{* *}$ & $4.39^{* *}$ \\
\hline
\end{tabular}

Significant differences with controls calculated by paired sample $t$-test. ${ }^{*}$ means significant at $P<0.05,{ }^{* *}$ means highly significant at $P<0.01$, ${ }^{* * *}$ means very high significant at $P<0.001$, and NS means nonsignificant.

TABLE 4: Effect of oat bran and barley bran supplementation for 8 weeks on antioxidant and oxidant enzymes in rats with induced hypercholesterolemia.

\begin{tabular}{|c|c|c|c|c|c|}
\hline Parameters & Statistics & G1 & G2 & G3 & G4 \\
\hline \multirow{2}{*}{ GST U/g.tissue } & Mean \pm SE & $24.10 \pm 0.26$ & $22.20 \pm 0.21$ & $23.86 \pm 1.23$ & $25.38 \pm 0.35$ \\
\hline & $t$-test & & $12.52^{* * *}$ & $0.06 \mathrm{NS}$ & $-0.60 \mathrm{NS}$ \\
\hline \multirow{2}{*}{ Catalase U/g.tissue } & Mean \pm SE & $164.44 \pm 3.03$ & $158.10 \pm 2.42$ & $173.32 \pm 1.70$ & $176.12 \pm 5.71$ \\
\hline & $t$-test & & $5.27^{* * *}$ & $-7.20^{* * *}$ & $-8.46 \mathrm{NS}^{* * *}$ \\
\hline \multirow{2}{*}{ Lipid peroxide nmol/g.tissue } & Mean \pm SE & $1057.40 \pm 71.72$ & $1058.30 \pm 1.79$ & $1001.80 \pm 73.90$ & $837.20 \pm 60.98$ \\
\hline & $t$-test & & $-0.01 \mathrm{NS}$ & $0.75 \mathrm{NS}$ & $3.62^{*}$ \\
\hline
\end{tabular}

Significant differences with controls calculated by paired sample $t$-test. ${ }^{*}$ means significant at $P<0.05,{ }^{* *}$ means highly significant at $P<0.01,{ }^{* * *}$ means very high significant at $P<0.001$, and NS means nonsignificant.

arrangement of uriniferous tubules and collecting tubules with glomerulus $(G)$ is observed. Figure $1(b)$ shows renal tissues of hypercholesterolemic group with bale renal tissues showing disrupted small uriniferous tubule and small Bowman's capsule with dilated urinary space. The renal tubules appeared irregular, dilated, and shrinkage of the glomerulus which led to a dilated urinary and collecting tubule space. Figure 1(c) shows renal tissues of oat bran-fed-group with convoluted uriniferous tubules with certain arranged collecting tubules. Figure 1(d) shows renal tissues of barley bran supplementation, the sections looked to be restoring the normal appearance, and the deposition of cholesterol was decreased in the kidney which showed well-defined urinary tubules and collecting tubules.

3.5.2. Liver. Hepatic tissues of control group with hepatic strands of cells and blood sinusoids are given in Figure 2(a). Figure 2(b) shows that the hepatic tissue suffering from hypercholesterolemia has fatty liver tissue with disrupted cells, vacuolated cytoplasm, and necrosis with disrupted hepatic strands. Figure 2(c) shows that the hepatic tissues of rats-fed oat bran restored the normal appearance of hepatic strands. Moreover, well-defined hepatic cords with polyhedral hepatocytes and normal appearing round nuclei are also shown. Figure 2(d) shows hepatic tissues of rats fed barley bran with no necrotic hepatic tissues with normal hepatic strands.

3.5.3. Testes. The testicular structure of the testes of control rats with normal and regular seminiferous tubules is shown in Figure 3(a). Figure 3(b) shows the hypercholesterolemic group suffering pathologic effects in which the structures of the seminiferous tubules were severely damaged and the thickness of the tubular walls was increased. The number of germinal cells was greatly decreased with a disturbance in their diameter. Figure 3(c) shows oat bran-fed group with normal and regular seminiferous tubules. The germinal cells were greatly regulated with normal diameter and mild dilatation of the seminiferous tubules with normal complete spermatogenic series with normal thickness. Figure 3(d) shows barley bran-fed group which has normal seminiferous tubules with normal germinal cell layers (arrows).

3.5.4. Heart. Cardiac tissues of control group showing normal structure of cardiac muscles consists of muscle fibers (arrows) are shown in Figure 4(a). Figure 4(b) shows cardiac tissues of hypercholesterolemia group showing increased hyalinization with cardiac muscles damage and necrosis of muscle fibers. Figure 4(c) shows cardiac tissues of rats fed oat bran showing minimal cardiac muscles damage with most normal tissues. Figure 4(d) shows cardiac tissues of barley bran-fed group showing disappearance of pathological changes with normal cardiac tissue.

\section{Discussion}

The present study has focused on testing and comparing the efficiency of two $\beta$-glucan containing nutrients, that is, oat bran and barley bran in ameliorating blood lipid profile. Large, prospective, epidemiologic studies proved a protective effect of dietary fiber against coronary heart disease through direct or indirect effects on serum lipids [30-32]. The reduction of cholesterol is possibly a sum of several effects; the most accepted one is due to decreased absorption of bile acids that causes a removal of steroids from the body by fecal 


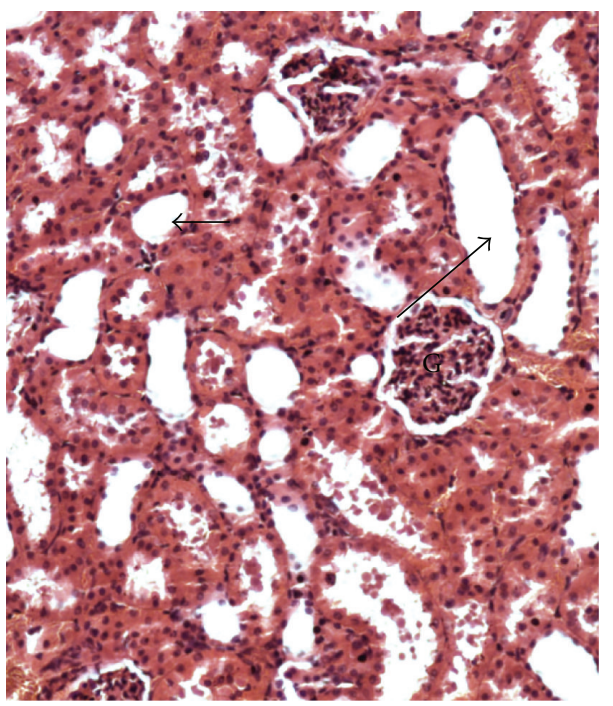

(a)

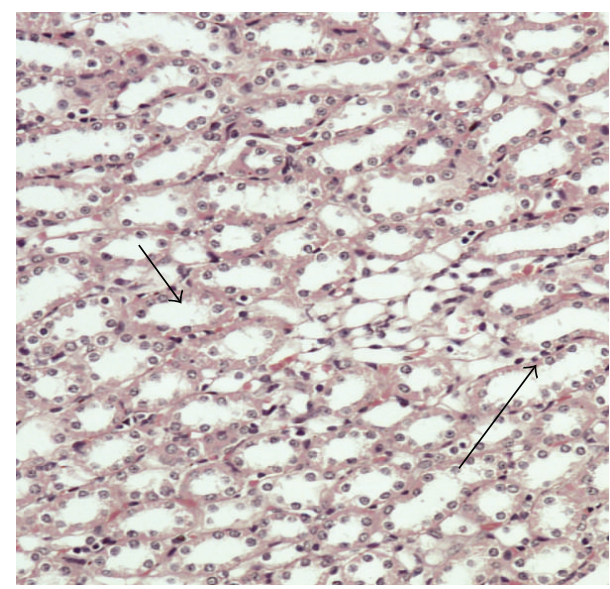

(c)

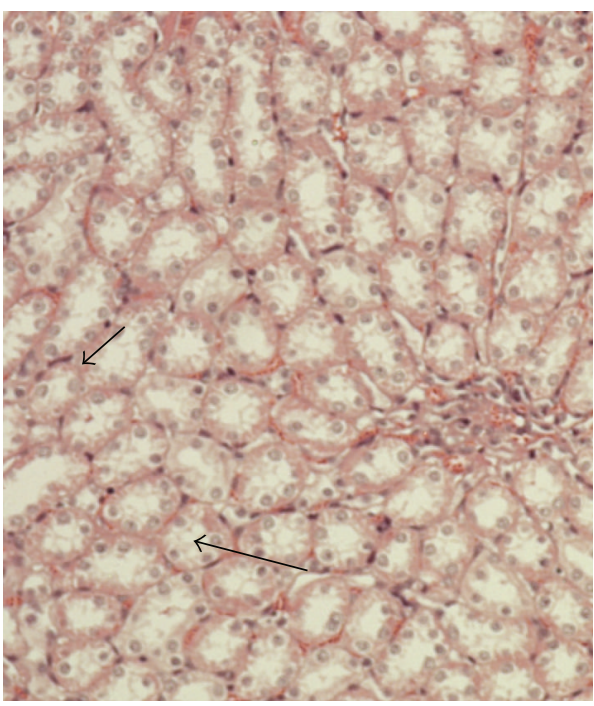

(b)

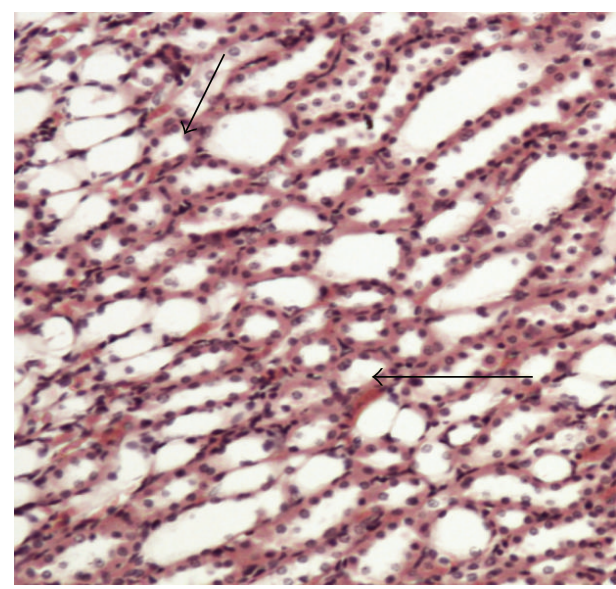

(d)

FIGURE 1: Effect of barley bran and oat bran supplementation for 2 months on renal tissue structure in rats with induced hypercholesterolemia. (a) Kidney renal tissues of control group showing normal renal structure with regulated nuclear arrangement of uriniferous tubules (small arrow) and collecting tubules (long arrow) with glomerulus (G), (b) renal tissues of hypercholesterolemic group showing bale renal tissues showing disrupted small uriniferous tubule (short arrow) and small Bowman's capsule with dilated urinary space. The renal tubules appeared irregular, dilated, and shrinkage of the glomerulus which led to dilated urinary and collecting tubule space (long arrow), (c) renal tissues of oat bran-treated group showing convoluted urineferous tubules (small arrow) with certain arranged collecting tubules (long arrow), and (d) renal tissues of barley bran supplementation: the sections looked to be restoring the normal appearance, and the deposition of cholesterol was decreased in the kidney which showed well-defined urinary tubules (short arrow) and collecting tubules (long arrow). $\times 200$ (H\&E stains).

excretion resulting in increased catabolism of cholesterol, an increase in the secretion of bile acids, a decrease in lipoprotein cholesterol secretion, and a reduction in the total body pool of cholesterol [33].

The current results showed that feeding hypercholesterolemic rats (supplemented $1 \%$ cholesterol in the diet) on $10 \%$ of barley bran or oat bran for eight weeks hindered significantly the rise of plasma lipids. This result is consistent with other investigations on animals and human [30-32], in terms of effect on total cholesterol, LDL-C, and triglyceride, whereas does not agree with the nonaffected HDL-C [30,34].
On the other hand, the current study showed that serum HDL was significantly increased with oat and barley bran. This result is consistent with that of Anderson [35] and disagrees with that of Braaten [36], who stated that oats $\beta$-glucan reduced the total and LDL-C level of hypercholesterolemic adults without changing HDL-C. Aly [11] reported that the supplementation of diet with either oat or wheat bran resulted in a significant decrease in the level of serum total lipid, total cholesterol, triglycerides, LDL-C, VLDL-C, and LDLC/HDL-C ratio with increase in the level of HDL-C compared with those fed high cholesterol. These results are concordant 


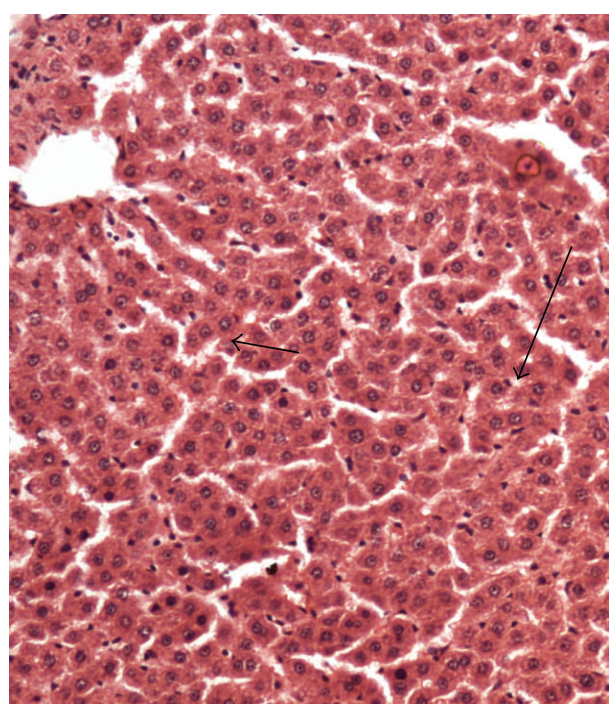

(a)

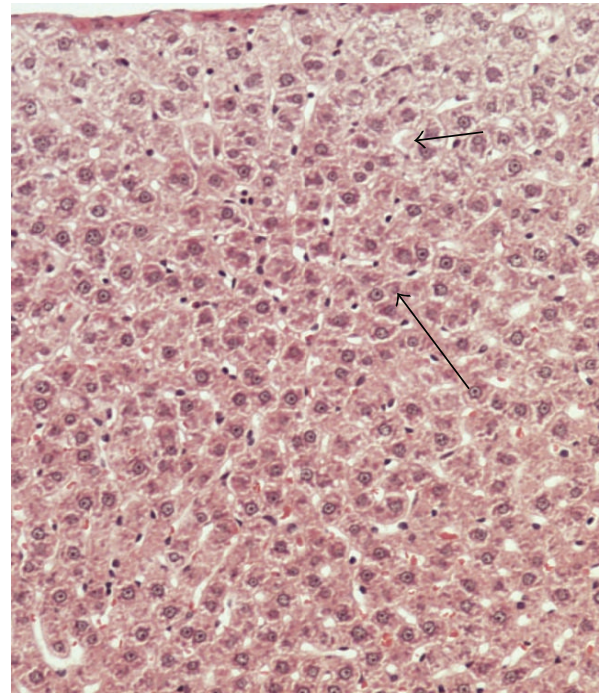

(c)

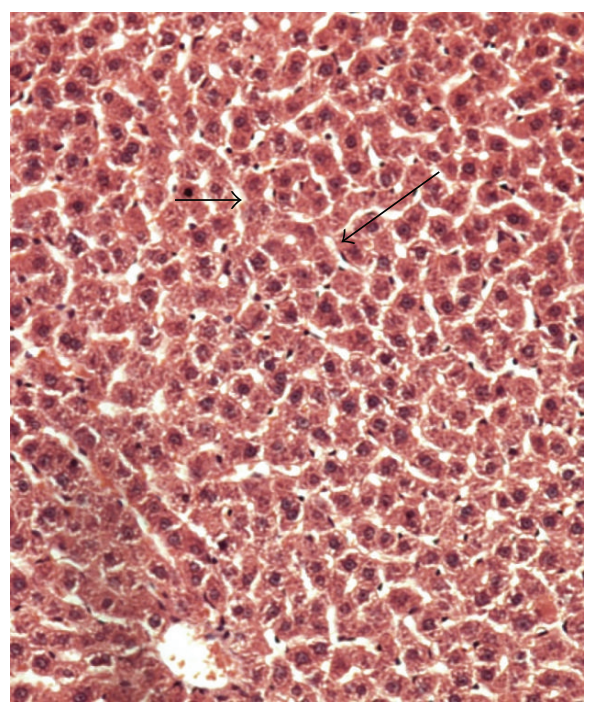

(b)

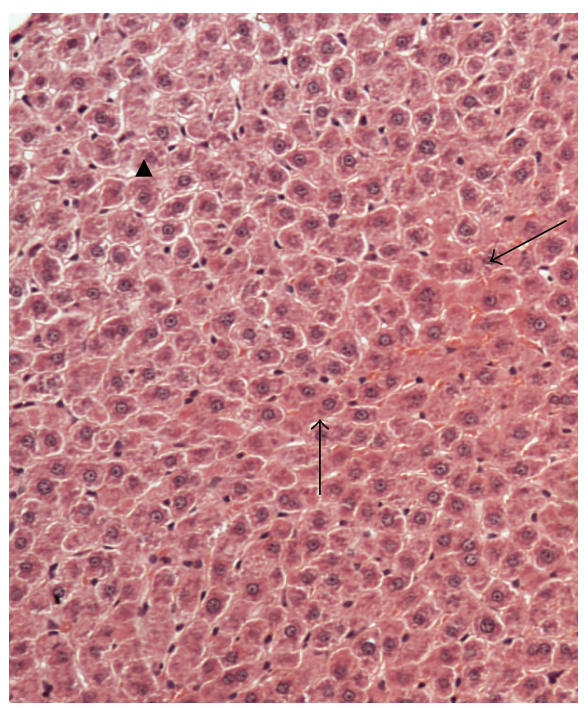

(d)

FIGURE 2: Effect of barley bran and oat bran supplementation for 2 months on liver tissue structure in rats with induced hypercholesterolemia. (a) Hepatic tissues of control group showing hepatic strands of cells (short arrow) blood sinusoids (long arrow), (b) hepatic tissue suffering hypercholesterolemia showing fatty liver tissue with disrupted cells, vacuolated cytoplasm, and necrosis with disrupted hepatic strands (arrows), (c) hepatic tissues pretreated oat bran shows be restoring the normal appearance of hepatic strands (short arrow), well-defined hepatic cords with polyhedral hepatocytes, and normal appearing round nuclei (long arrow), and (d) hepatic tissues pretreated barley bran shows no necrotic hepatic tissues with normal hepatic strands (arrows). $\times 200$ (H\&E stains).

with our results. It is worthy to mention that the current biochemical tests results revealed that oats had better effect in reducing the level of lipids than barley.

On the other hand the present results showed that, the increase in cholesterol in cholesterol-enriched diet resulted in a significant increase in total lipids, total cholesterol, triglycerides, LDL-C, VLDL-C, and LDL/HDL ratio accompanied with decrease in HDL-C, thus providing a model for dietary hyperlipidemia. These results are in concordance with other results, on animals with nutritionally induced hypercholesterolemia [37].

The current results showed a significant increase in the level of enzyme activity of ALT, AST, and ALP in rats' serum after induction of hypercholesterolemia, compared with those received basal diet. This result is consistent with Mahfouz and Kummerow [38].

The oral administration of $1 \%$ cholesterol in the diet to rats under study increased lipid peroxide (which is an oxidative stress biomarker) and decreased GST and catalase (which are antioxidant enzymes) in the kidney homogenate, compared with the negative control. The decrease in GST and catalase levels in the kidney homogenate of cholesterol-fed rats could have a protective role against oxidation, thus preventing the formation of lipid peroxidation. In contrast, feeding hypercholesterolemic rats (fed 1\% cholesterol in the diet) on diets containing $10 \%$ oat bran or barley bran showed a 


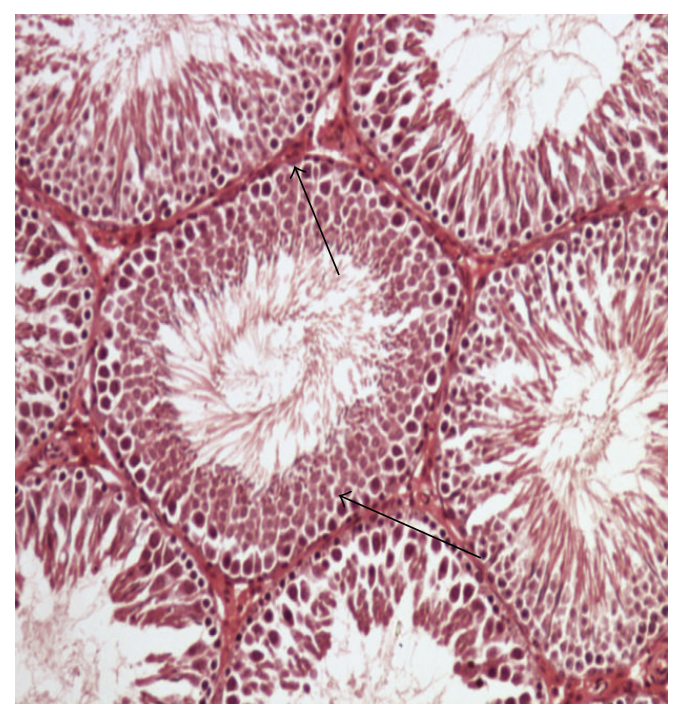

(a)

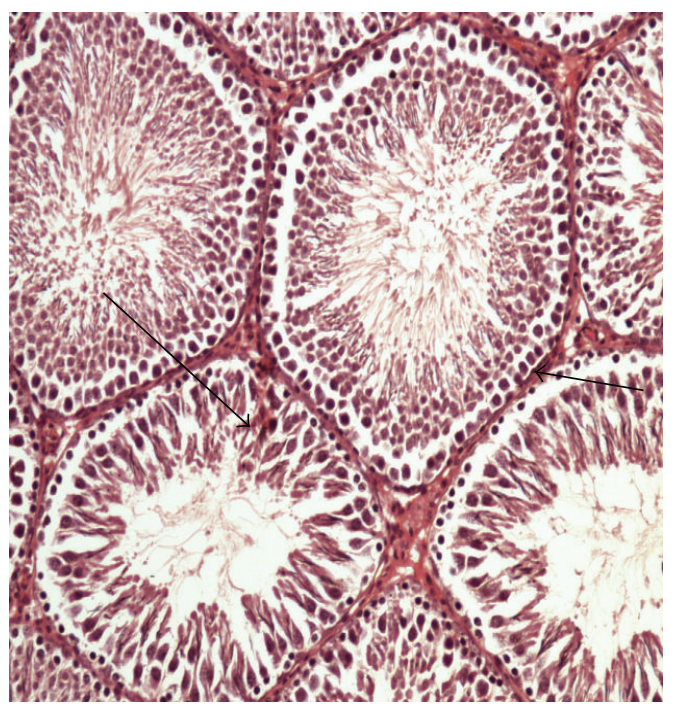

(c)

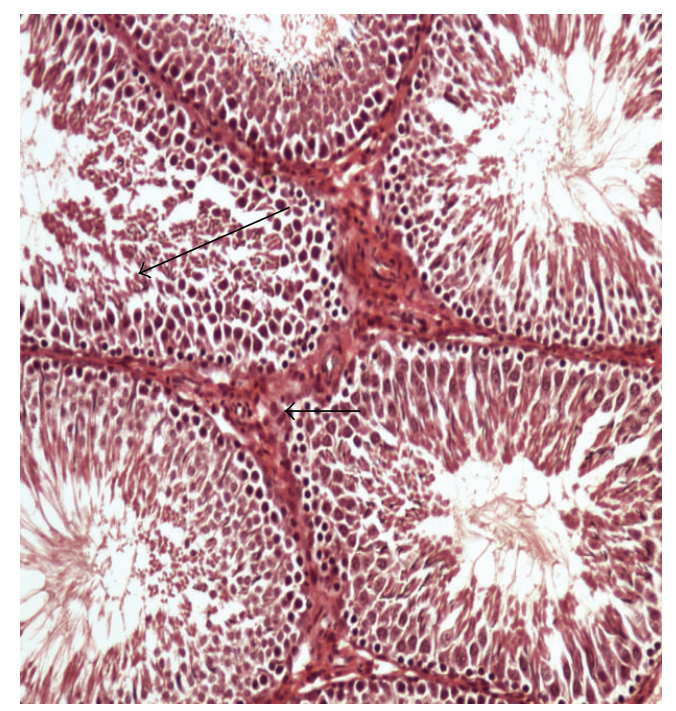

(b)

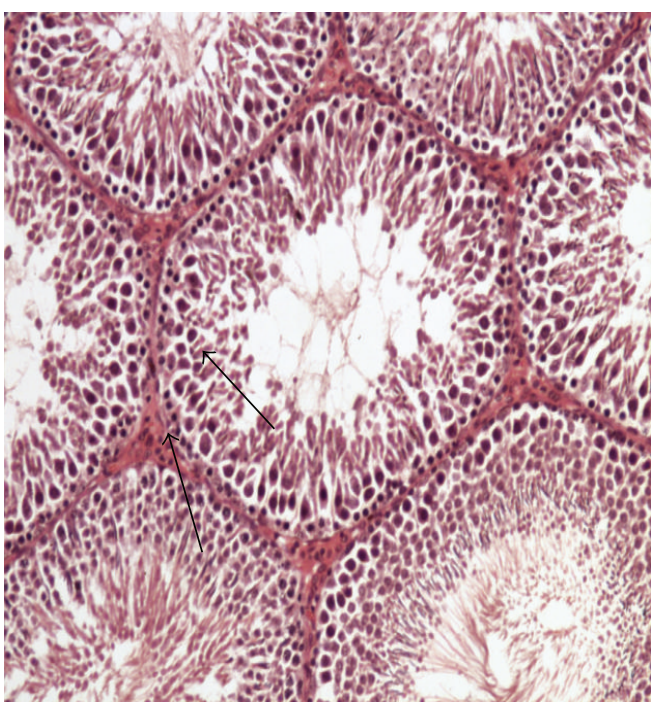

(d)

FIGURE 3: Effect of barley bran and oat bran supplementation for 2 months on testicular structure in rats with induced hypercholesterolemia. (a) Control rats showing normal and regular seminiferous tubules (arrows), (b) hypercholesterolemia group showing the pathologic effects in which the structures of the seminiferous tubules were severely damaged, and thickness of the tubular walls was increased (short arrow) and number of germinal cells was greatly decreased with a disturbance in their diameter (long arrow), (c) oat bran-treated group showing normal and regular seminiferous tubules, and germinal cells were greatly regulated with normal diameter and mild dilatation of the seminiferous tubules with normal complete spermatogenic series (short arrow) with normal thickness (long arrow), and (d) barley bran-treated group showing normal seminiferous tubules with normal germinal cell layers (arrows). $\times 400$ (H\&E Stain).

significant decrease in the level of lipid peroxide and increase in the level of antioxidant GST and catalase compared with those received only $1 \%$ cholesterol (G2). These results are consistent with other results suggesting protective role of dietary fiber [11, 38, 39].

The current investigation showed a significant increase in the level of creatinine, uric acid, and urea compared with that of the negative control. This result is consistent with other studies demonstrated a relationship between kidney disease and increased cholesterol in the diet [40-42]. In contrast, the rats fed oat bran and barley bran showed significant decrease in the level of uric acid, urea, and creatinine compared with the positive control. This result agrees with the assumption that dietary fiber improves the level of kidney function [43].

The histopathological investigations showed histological alteration in the target organs (kidney, liver, testes, and heart) in the $1 \%$ cholesterol-fed group (G2). This result supported with previous studies suggesting a correlation between hypercholesterolemia and histological changes in the organs [4446]. On the other hand, improvement in microscopic examination of tissues in groups fed on either oat bran or barley bran together with cholesterol. Both oats bran and barley bran 


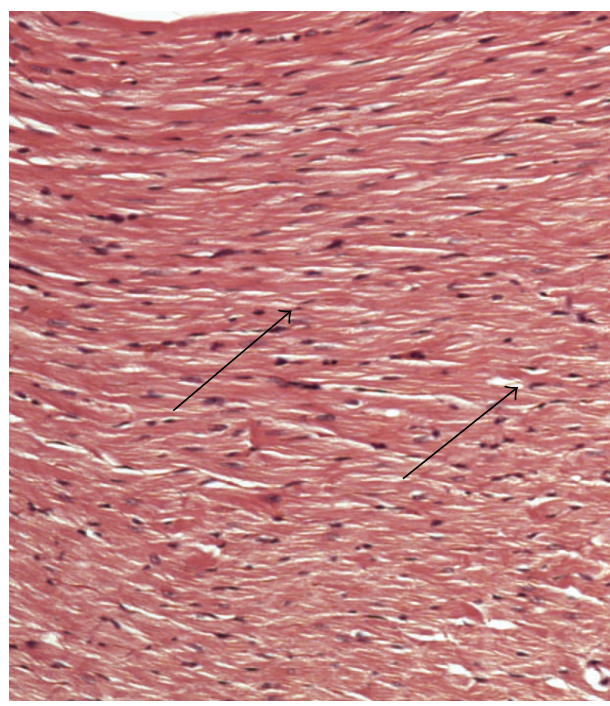

(a)

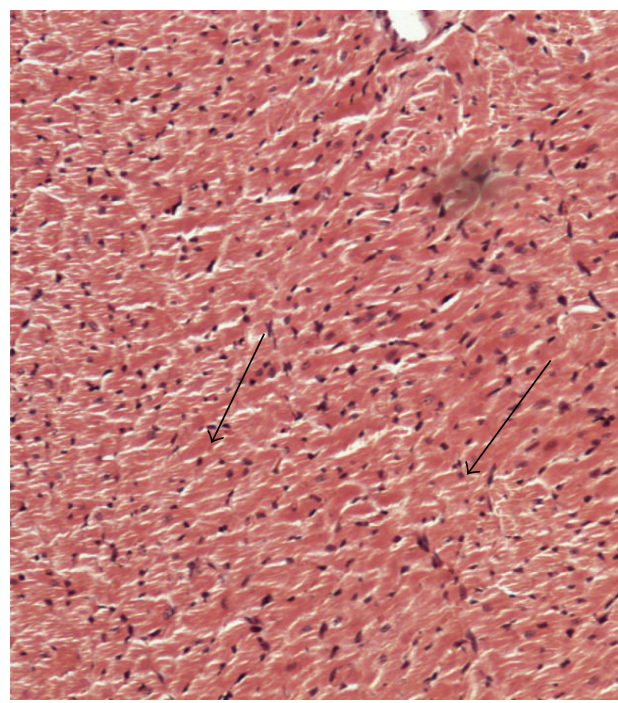

(c)

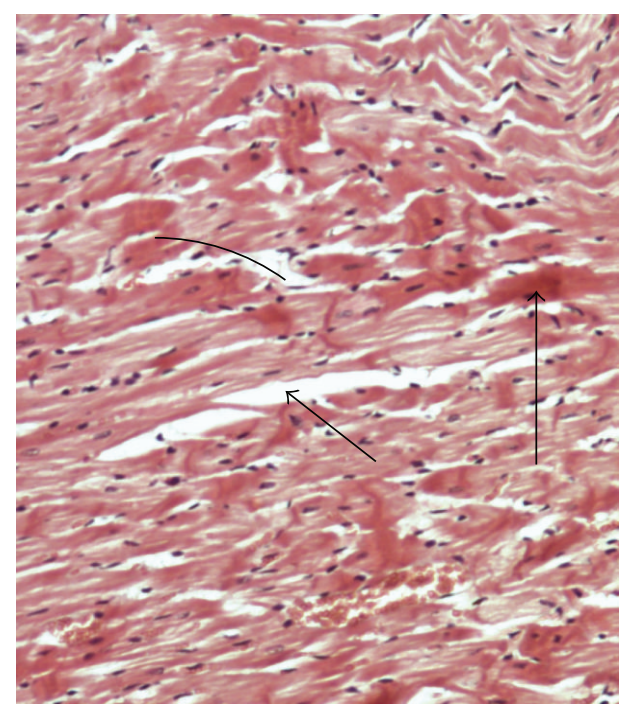

(b)

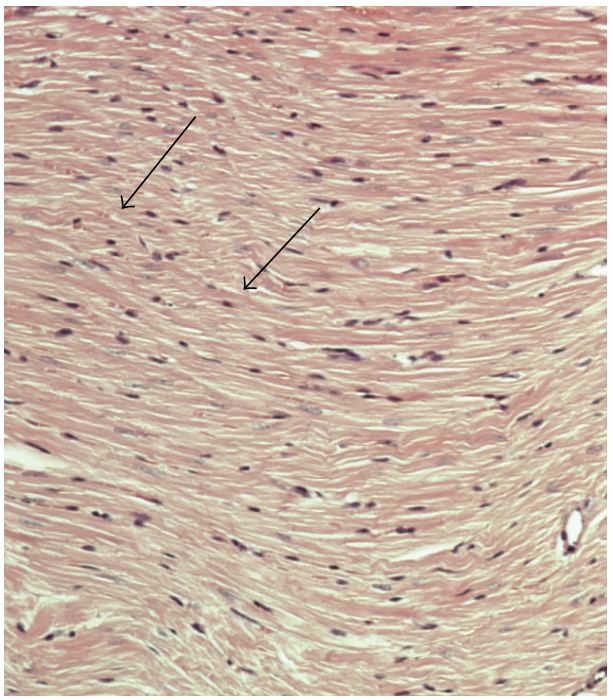

(d)

Figure 4: (a) Cardiac tissues of control group showing normal structure of cardiac muscles consist of muscle fibers (arrows), (b) cardiac tissues of hypercholesterolemia group showing increased hyalinization (short arrow) with cardiac muscles damage (long arrow) and necrosis of muscle fibers (curved arrow), (c) cardiac tissues pretreated with oat bran shows minimal cardiac muscles damage with most normal tissues (arrows), and (d) pretreated with barley bran showing disappearance of pathological changes with normal cardiac tissue (arrows). $\times 200$ (H\&E stains).

have a protective role against these histological alterations due to their higher content of antioxidant substance mainly beta-glucan which has powerful antioxidant attributes, as its molecules help to prevent cell damage, working in association with enzymes and reduces the effect of dietary cholesterol resulting in the increase in the GSH product by the organs [47].

It could be concluded that both oat bran and barley bran succeeded in lowering the lipid profile levels in the blood of hypercholesterolemic rats. In addition, oat bran appeared more efficient than barley bran as revealed by the different biochemical and histological investigations. The findings of our study support the addition of the barley and oat bran to human meal in order to ameliorate the blood lipid profile.

\author{
Abbreviations \\ CBC: Complete blood count \\ CHD: Coronary heart diseases \\ EDTA: Ethylenediaminetetraacetic acid \\ G: Glomerulus \\ G1: $\quad$ Fed normal diet \\ G2: $\quad$ Fed 1.0\% w/w cholesterol in the diet \\ G3: $\quad$ Fed $1.0 \% \mathrm{w} / \mathrm{w}$ cholesterol and supplemented \\ with $10 \%$ oat bran \\ G4: $\quad$ Fed $1.0 \% \mathrm{w} / \mathrm{w}$ cholesterol as in (G2) and \\ supplemented with $10 \%$ barley bran \\ GST: Glutathione S-transferase \\ H\&S: Hematoxylin and eosin
}


LDL: Low density lipoproteins

LDL-C: Low density lipoproteins cholesterol

s.LDL-C: Serum low density lipoproteins cholesterol

s.TC: $\quad$ Serum total cholesterol

s.TG: $\quad$ Serum triglyceride

s.VLDL-C: Serum very low density lipoprotein-cholesterol

SE: $\quad$ Standard errors

SPSS: $\quad$ Statistical Program for Sociology Scientists

TC: $\quad$ Total cholesterol.

\section{Conflict of Interests}

The authors of this paper have no conflict of interests.

\section{Acknowledgment}

Thanks are due to King Abdul-Aziz City for Science and Technology for financial support for this research, Grant no. (11-0760).

\section{References}

[1] F. M. Sacks, M. A. Pfeffer, L. A. Moye et al., "The effect of pravastatin on coronary events after myocardial infarction in patients with average cholesterol levels," The New England Journal of Medicine, vol. 335, no. 14, pp. 1001-1009, 1996.

[2] P. S. Nijjar, F. M. Burke, A. Bloesch, and D. J. Rader, "Role of dietary supplements in lowering low-density lipoprotein cholesterol: a review," Journal of Clinical Lipidology, vol. 4, no. 4, pp. 248-258, 2010.

[3] K. M. Behall, D. J. Scholfield, and J. Hallfrisch, "Lipids significantly reduced by diets containing barley in moderately hypercholesterolemic men," Journal of the American College of Nutrition, vol. 23, no. 1, pp. 55-62, 2004.

[4] M. H. Alu'datt, T. Rababah, K. Ereifej et al., "Effects of barley flour and barley protein isolate on chemical, functional, nutritional and biological properties of Pita bread," Food Hydrocolloids, vol. 26, no. 1, pp. 135-143, 2012.

[5] P. Sullivana, E. Arendt, and E. Gallaghera, "The increasing use of barley and barley by-products in the production of healthier baked goods," Trends in Food Science \& Technology, vol. 29, pp. 124-134, 2013.

[6] S. R. Glore, D. Van Treeck, A. W. Knehans, and M. Guild, "Soluble fiber and serum lipids: a literature review," Journal of the American Dietetic Association, vol. 94, no. 4, pp. 425-436, 1994.

[7] A. S. Truswell, "Dietary fibre and plasma lipids," European Journal of Clinical Nutrition, vol. 49, no. 3, pp. S105-S109, 1995.

[8] Y. Malkki, K. Pelkonen, O. Myllymaki, R. Torronen, O. Hanninen, and K. Syrjanen, "Effects of oat bran concentrate on rat serum lipids and liver fat infiltration," European Journal of Clinical Nutrition, vol. 49, no. 3, pp. S321-S324, 1995.

[9] E. Naumann, A. B. Van Rees, G. Önning, R. Öste, M. Wydra, and R. P. Mensink, " $\beta$-Glucan incorporated into a fruit drink effectively lowers serum LDL-cholesterol concentrations," American Journal of Clinical Nutrition, vol. 83, no. 3, pp. 601-605, 2006.

[10] D. A. J. M. Kerckhoffs, G. Hornstra, and R. P. Mensink, "Cholesterol-lowering effect of $\beta$-glucan from oat bran in mildly hypercholesterolemic subjects may decrease when $\beta$-glucan is incorporated into bread and cookies," American Journal of Clinical Nutrition, vol. 78, no. 2, pp. 221-227, 2003.
[11] N. H. Aly, "Effect of dietary oat and wheat bran on biochemical changes in rats fed high fat-high cholesterol diets," Journal of Applied Sciences Research, vol. 8, pp. 598-604, 2012.

[12] J. Chen, J. He, R. P. Wildman, K. Reynolds, R. H. Streiffer, and P. K. Whelton, "A randomized controlled trial of dietary fiber intake on serum lipids," European Journal of Clinical Nutrition, vol. 60, no. 1, pp. 62-68, 2006.

[13] D. G. Stevenson, G. E. Inglett, D. Chen, A. Biswas, F. J. Eller, and R. L. Evangelista, "Phenolic content and antioxidant capacity of supercritical carbon dioxide-treated and air-classified oat bran concentrate microwave-irradiated in water or ethanol at varying temperatures," Food Chemistry, vol. 108, no. 1, pp. 23-30, 2008.

[14] K. E. Charlton, L. C. Tapsell, M. J. Batterham et al., "Effect of 6 weeks' consumption of $\beta$-glucan-rich oat products on cholesterol levels in mildly hypercholesterolaemic overweight adults," British Journal of Nutrition, vol. 1, pp. 1-11, 2011.

[15] R. A. Othman, M. H. Moghadasian, and P. J. H. Jones, "Cholesterol-lowering effects of oat $\beta$-glucan," Nutrition Reviews, vol. 69, no. 6, pp. 299-309, 2011.

[16] J. M. Keenan, M. Goulson, T. Shamliyan, N. Knutson, L. Kolberg, and L. Curry, "The effects of concentrated barley $\beta$ glucan on blood lipids in a population of hypercholesterolaemic men and women," British Journal of Nutrition, vol. 97, no. 6, pp. 1162-1168, 2007.

[17] J. J. Pins and H. Kaur, "A review of the effects of barley $\beta$-glucan on cardiovascular and diabetic risk," Cereal Foods World, vol. 51, no. 1, pp. 8-11, 2006.

[18] P. Åman, "Cholesterol-lowering effects of barley dietary fibre in humans: scientific support for a generic health claim," Scandinavian Journal of Food and Nutrition, vol. 50, no. 4, pp. 173-176, 2006.

[19] S. Araki, K. Ito, S. Aoe, and S. Ikegami, "Physiological functions of barley and the health claims on food labels," Japanese Journal of Nutrition and Dietetics, vol. 67, pp. 235-251, 2009.

[20] American Institute of Nutrition, "Second report of AIN Ad Hoc Committee on standards for nutritional studies," Journal of Nutrition, vol. 110, article 1726, 1980.

[21] Z. Moussavi, M. O. Plancke, O. P. Olivier Ph., D. Lesieur, J. C. Fruchart, and J. Sauzieres, "Lipid lowering actions of 7-(2methylene butyryl)-( $2 \mathrm{H})-1,4$-benzoxazin-3-(4H)-one derivatives in mice, rats and Syrian hamsters," Clinica Chimica Acta, vol. 180, no. 1, pp. 35-44, 1989.

[22] F. L. Shinnick, M. J. Longacre, S. L. Ink, and J. A. Marlett, "Oat fiber: composition versus physiological function in rats," Journal of Nutrition, vol. 118, no. 2, pp. 144-151, 1988.

[23] R. C. Jain, H. Khubnani, and A. Khubnani, "Action of garlic on the hyperlipidemia of experimental induced immune complex disease in rabbits," Indian Journal of Pharmacology, vol. 29, no. 4, pp. 255-257, 1997.

[24] O. M. Ahmed, "Histopathological and biochemical evaluation of Liver and kidney lesions in streptozotocin diabetic rats treated with glimepiride and various plant extracts," Journal of Union of Arab Biologists Cairo, vol. 164, pp. 585-625, 2001.

[25] H. B. Waynforth, Experimental Animal and Surgical Technique in the Rat, Academic Press, New York, NY, USA, 1980.

[26] W. Habig, M. Pabst, and W. J. Jakoby, "Intestinal glutathione S-transferase activity in flounder Platichthys flesus collected from contaminated and reference sites," The Journal of Biological Chemistry, vol. 249, pp. 7130-77139, 1974.

[27] H. Aebi, "Catalase in vitro," Methods in Enzymology, vol. 105, no. C, pp. 121-126, 1984. 
[28] H. Ohkawa, N. Ohishi, and K. Yagi, "Assay for lipid peroxides in animal tissues by thiobarbituric acid reaction," Analytical Biochemistry, vol. 95, no. 2, pp. 351-358, 1979.

[29] R. A. Drury, E. A. Wallington, and R. Cancerson, Carlton's histopathological techniques, Oxford University Press, Oxford, UK, 4th edition, 1976.

[30] R. K. Newman, C. W. Newman, and H. Graham, "The hypocholesterolemic function of barley $\beta$-glucans," Cereal Foods World, vol. 34, article 883, 1989.

[31] J. Czerwiński, E. Bartnikowska, H. Leontowicz et al., "Oat (Avena sativa L.) and amaranth (Amaranthus hypochondriacus) meals positively affect plasma lipid profile in rats fed cholesterol-containing diets," Journal of Nutritional Biochemistry, vol. 15, no. 10, pp. 622-629, 2004.

[32] K. M. Queenan, M. L. Stewart, K. N. Smith, W. Thomas, R. G. Fulcher, and J. L. Slavin, "Concentrated oat $\beta$-glucan, a fermentable fiber, lowers serum cholesterol in hypercholesterolemic adults in a randomized controlled trial," Nutrition Journal, vol. 6, article 6, 2007.

[33] Y. Malkki, S. S. Cho, and M. L. Dreher, "Oat fiber: production, composition, physicochemical properties, physiological effects, safety, and food applications," in Handbook of Dietary Fiber, pp. 497-517, 2001.

[34] G. F. Keogh, G. J. S. Cooper, T. B. Mulvey et al., "Randomized controlled crossover study of the effect of a highly $\beta$-glucanenriched barley on cardiovascular disease risk factors in mildly hypercholesterolemic men," American Journal of Clinical Nutrition, vol. 78, no. 4, pp. 711-718, 2003.

[35] J. W. Anderson, "Dietary fiber and diabetes," in Medical Aspects of Dietary Fiber, G. A. Spiller and R. M. Kay, Eds., pp. 193-221, Plenum Medical, New York, NY, USA, 1980.

[36] J. T. Braaten, P. J. Wood, F. W. Scott et al., "Oat $\beta$-glucan reduces blood cholesterol concentration in hypercholesterolemic subjects," European Journal of Clinical Nutrition, vol. 48, no. 7, pp. 465-474, 1994.

[37] T. Kishida, H. Nogami, H. Ogawa, and K. Ebihara, "The hypocholesterolemic effect of high amylose cornstarch in rats is mediated by an enlarged bile acid pool and increased fecal bile acid excretion, not by cecal fermented products," Journal of Nutrition, vol. 132, no. 9, pp. 2519-2524, 2002.

[38] M. M. Mahfouz and F. A. Kummerow, "Cholesterol-rich diets have different effects on lipid peroxidation, cholesterol oxides, and antioxidant enzymes in rats and rabbits," Journal of Nutritional Biochemistry, vol. 11, no. 5, pp. 293-302, 2000.

[39] J. Balkan, S. Doğru-Abbasoǧlu, G. Aykaç-Toker, and M. Uysal, "The effect of a high cholesterol diet on lipids and oxidative stress in plasma, liver and aorta of rabbits and rats," Nutrition Research, vol. 24, no. 3, pp. 229-234, 2004.

[40] E. S. Schaeffner, T. Kurth, G. C. Curhan et al., "Cholesterol and the risk of renal dysfunction in apparently healthy men," Journal of the American Society of Nephrology, vol. 14, no. 8, pp. 20842091, 2003.

[41] R. Trevisan, A. R. Dodesini, and G. Lepore, "Lipids and renal disease," Journal of the American Society of Nephrology, vol. 17, no. 2, pp. S145-S147, 2006.

[42] M. K. Abdel-Rahman, "Influence of dietary fat on renal function, lipid profile, sex hormones, and electrolyte balance in rats," European Journal of Lipid Science and Technology, vol. 112, no. 10, pp. 1166-1172, 2010.

[43] D. S. Rampton, S. L. Cohen, and V. B. De Crammond, "Treatment of chronic renal failure with dietary fiber," Clinical Nephrology, vol. 21, no. 3, pp. 159-163, 1984.
[44] M. E. Altunkaynak, E. Özbek, B. Z. Altunkaynak, I. Can, D. Unal, and B. Unal, "The effects of high-fat diet on the renal structure and morphometric parametric of kidneys in rats," Journal of Anatomy, vol. 212, no. 6, pp. 845-852, 2008.

[45] J. Dimitrova-Shumkovska, L. Veenman, T. Ristoski, S. Leschiner, and M. Gavish, "Chronic high fat, high cholesterol supplementation decreases $18 \mathrm{kDa}$ Translocator Protein binding capacity in association with increased oxidative stress in rat liver and aorta," Food and Chemical Toxicology, vol. 48, no. 3, pp. 910921, 2010.

[46] A. Ouvrier, G. Alves, C. Damon-Soubeyrand et al., "Dietary cholesterol-induced post-testicular infertility," PLoS ONE, vol. 6, no. 11, Article ID e26966, 2011.

[47] H. Sekena, M. A. Abdel-Wahab, A. M. Mahmoud, and M. Azza, "Chemoprevention of Barley and Sage against acrylamideInduced genotoxic, biochemical and histopathological alterations in rats," Egyptian Journal of Hospital Medicine, vol. 15, pp. 40-56, 2004. 

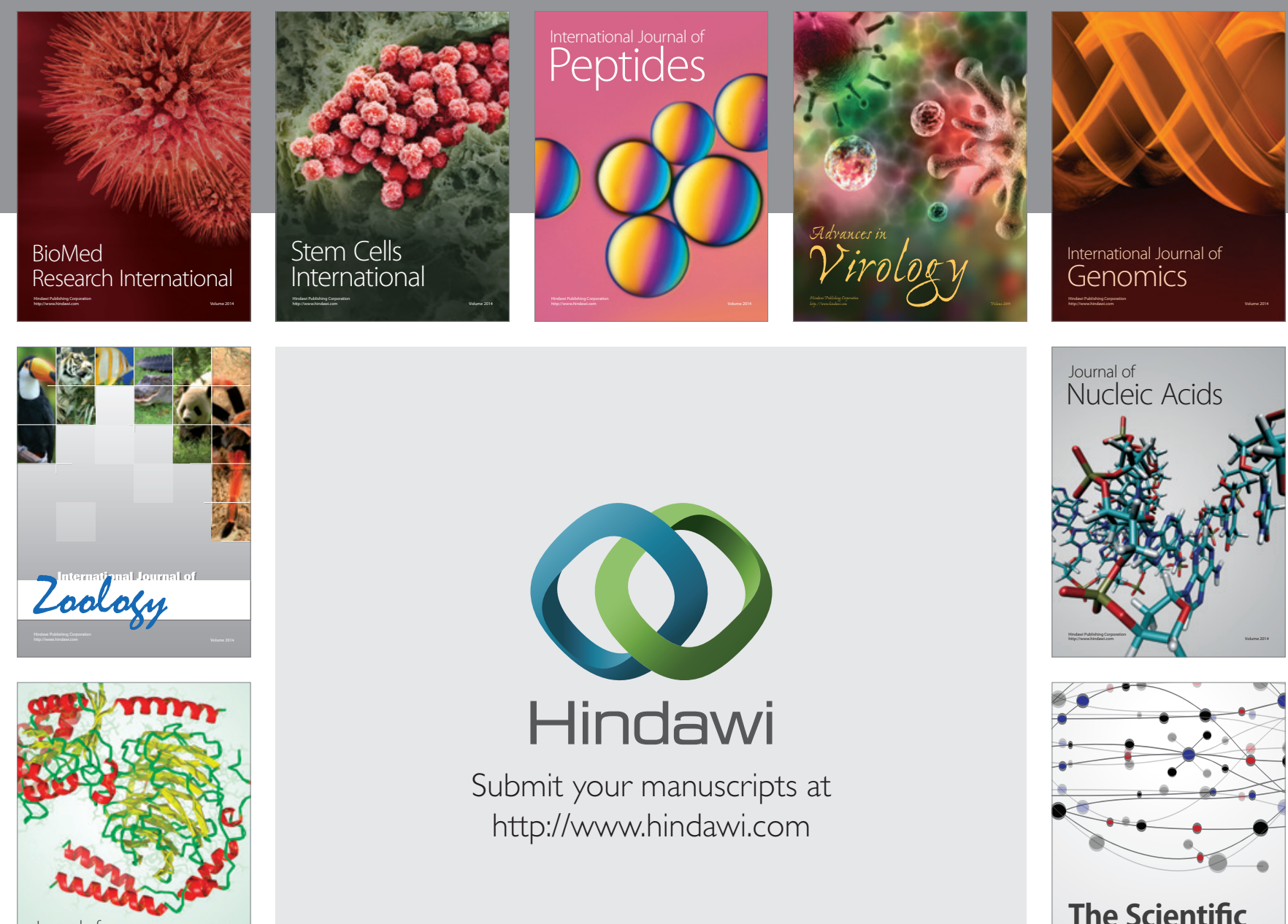

Submit your manuscripts at

http://www.hindawi.com

Journal of
Signal Transduction
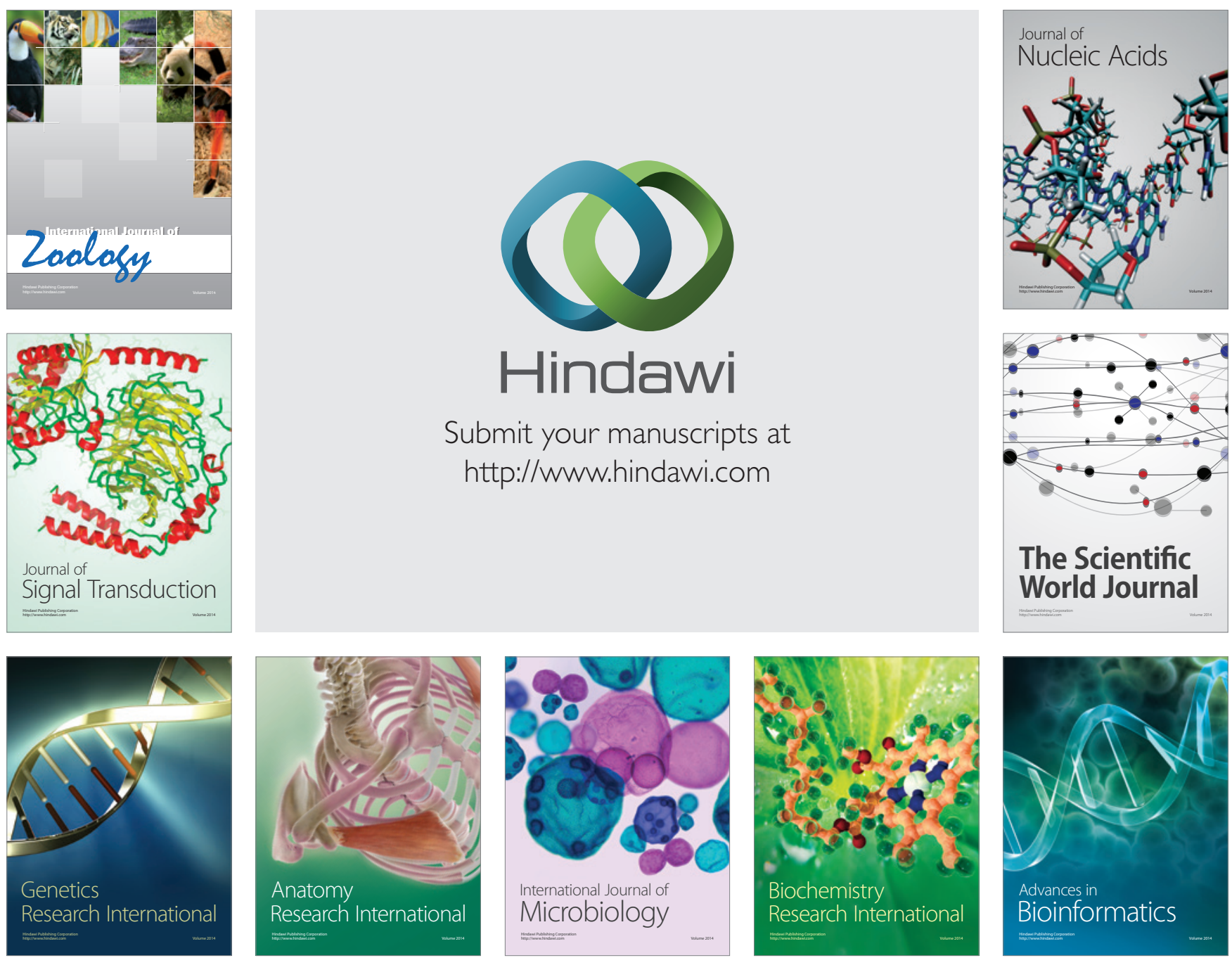

The Scientific World Journal
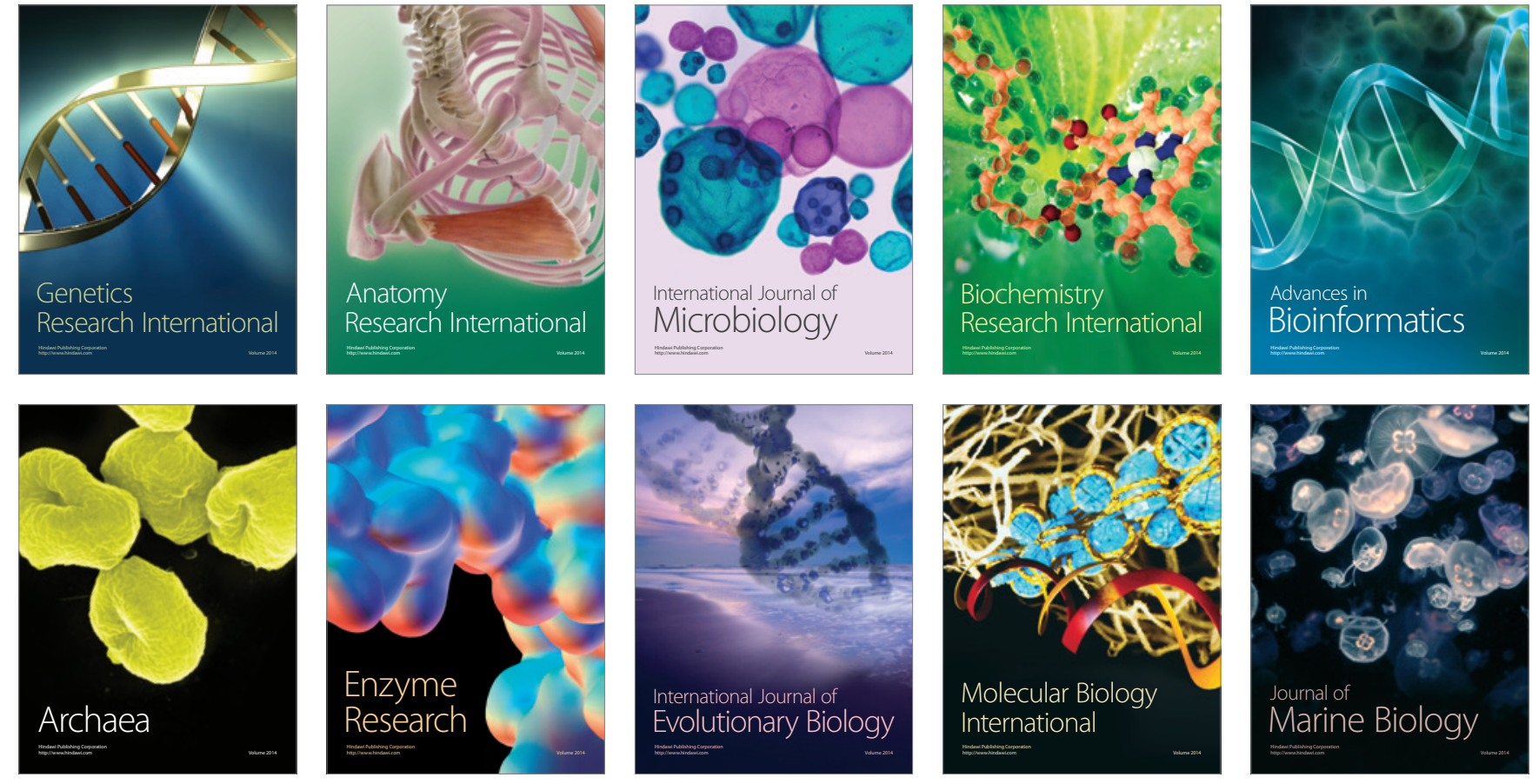